\title{
Ion irradiation of the Murchison meteorite: Visible to mid-infrared spectroscopic results
}

\author{
C. Lantz ${ }^{1,2}$, R. Brunetto ${ }^{3}$, M. A. Barucci ${ }^{1}$, E. Dartois ${ }^{3}$, J. Duprat ${ }^{4}$, C. Engrand ${ }^{4}$, M. Godard ${ }^{4}$, D. Ledu ${ }^{4}$, and E. Quirico ${ }^{5}$
}

${ }^{1}$ Laboratoire d'Études Spatiales et d'Instrumentation en Astrophysique (LESIA) - Observatoire de Paris, CNRS (UMR 8109)/UPMC (Paris 6) / Univ. Paris Diderot (Paris 7), 92195 Meudon Cedex, France e-mail: cateline.lantz@obspm. fr

2 Université Paris Diderot, Sorbonne Paris Cité, 75205 Paris Cedex 13, France

3 Institut d'Astrophysique Spatiale (IAS), CNRS (UMR 8617)/Université Paris-Sud (Paris 11), 91405 Orsay Cedex, France

4 Centre de Sciences Nucléaires et de Sciences de la Matière (CSNSM), IN2P3 - CNRS (UMR 8609)/Université Paris-Sud (Paris 11), 91405 Orsay Cedex, France

5 Institut de Planétologie et d'Astrophysique de Grenoble (IPAG), Université J. Fournier - Grenoble 1/CNRS-INSU (UMR 5274), 38041 Grenoble Cedex 9, France

Received 24 November 2014 / Accepted 3 March 2015

ABSTRACT

\begin{abstract}
Aims. The goal of this study is to simulate space weathering processes on primitive bodies. We use ion implantation as a simulation of solar wind irradiation, which has been suggested by several authors to be the major component of space weathering on main belt asteroids. The laboratory analogs we irradiate and analyze are carbonaceous chondrites; we started the study with the Allende CV meteorite and in this companion paper we present results on the Murchison CM meteorite.

Methods. We performed irradiations on pressed pellets of Murchison with $40 \mathrm{keV} \mathrm{He}^{+}$and $\mathrm{Ar}^{+}$ions using fluences up to $3 \times$ $10^{16}$ ions $/ \mathrm{cm}^{2}$. Reflectance spectra were acquired ex situ before and after irradiation in the visible to mid-infrared range $(0.4-16 \mu \mathrm{m})$. A Raman analysis was also performed to investigate the modifications of the aromatic carbonaceous component.

Results. Our results indicate that spectral variations after irradiation within the visible range are smaller than spectral variations due to sample grain size or viewing geometry of the Murchison meteorite. The aqueous alteration band profile near $3 \mu \mathrm{m}$ changes after irradiation, as adsorbed water is removed, and phyllosilicates are affected. Raman spectroscopy highlights the insoluble organic matter (IOM) modification under irradiation. We observe a shift of the silicates band at $9.9 \mu \mathrm{m}$, probably due to a preferential loss of $\mathrm{Mg}$ (compared to $\mathrm{Fe}$, the lighter $\mathrm{Mg}$ is more easily sputtered backward) and/or amorphization of $\mathrm{Mg}$-rich materials. We compare our results to previous experiments on organic-rich materials (like asphaltite or carbonaceous chondrites), and on ordinary chondrites and olivine grains. We find that the reddening/darkening trend observed on silicate-rich surfaces is not valid for all carbonaceous chondrites, and that the spectral modifications after irradiation are a function of the initial albedo.
\end{abstract}

Key words. techniques: spectroscopic - meteorites, meteors, meteoroids - minor planets, asteroids: general

\section{Introduction}

The space weathering ( $\mathrm{SpWe}$ ) processes, i.e., particle irradiation and impact leading to surface alteration of atmosphere-less bodies in the solar system, have been unevenly studied for S- and C-complex asteroids. This disparity can be explained because SpWe was first discovered on the Moon and then applied to the abundant ordinary chondrites (OCs) that are linked to the S-type asteroids, which are easier to observe thanks to a relatively high albedo. Two of these near-Earth bodies have been visited by space missions, NEAR-Shoemaker on 433 Eros and Hayabusa on 25143 Itokawa. The well-known spectral reddening and darkening trends have been observed in the visible-NIR range (see Clark et al. 2001; Ishiguro et al. 2007, respectively), most probably as a consequence of the formation of finely dispersed opaque minerals (Noble et al. 2007). This has been confirmed by laboratory analysis of Itokawa grains (Noguchi et al. 2011) brought back to Earth thanks to the sample return Hayabusa mission. The nanophase reduced iron $(\mathrm{npFe} 0)$ are the compounds that have been suggested as the major cause of SpWe spectral effects on OCs, that is to say the reddening and darkening of the
VIS-NIR spectrum (see review in Clark et al. 2002). The effects of the npFe0 have been modeled analytically by Hapke (2001).

However, OC-like material represents only a small fraction of the asteroid belt. Half of the main belt asteroids belong to the primitive (low albedo, rich in organic compounds) C-, P-, D-, B-, F-, and G-types (Gaffey et al. 2002; DeMeo \& Carry 2014), while only $5 \%$ of meteorites are carbonaceous chondrites (CCs, the meteoritic analogs of some classes of primitive asteroids; Grady 2000), probably because of large biases influencing the meteorite collections. So far, we have few clues to how SpWe acts on primitive bodies and if it is the same that acts on S-types, i.e., dominant effects from npFe0 formation. Predictions for these bodies suggested a possible limited effect on the spectral slope (Pieters et al. 2000), or even an opposite effect defined as blueing of the slope (Nesvorný et al. 2005). Contradictory results have also been obtained in laboratory SpWe ion irradiation experiments performed on primitive materials: spectral reddening on Allende (Lazzarin et al. 2006; Brunetto et al. 2014) and Frontier Mountain 95002 (Lazzarin et al. 2006), and blueing on asphaltite (Moroz et al. 2004a) and Tagish Lake meteorite (Vernazza et al. 2013) have been observed. Tagish Lake also shows a significant spectral flattening after pulse laser irradiation 
(Hiroi et al. 2013). The CM Mighei meteorite was studied after laser treatment as a simulation of the micrometeoritic impact melting, and spectral brightening and reddening (in the whole VIS-NIR range) were observed (Moroz et al. 2004b). It is interesting to note that if one focuses on the visible range, the result of the experiment is a slight blueing. Scanning electron microscopy study (Shingareva et al. 2004) showed that the laser treatment mostly resulted in the formation of spherical glassy droplets that contain Fe-rich inclusions. The same conclusions have been made on synthetic analogs of CM chondrites by Shingareva et al. (2003).

Space weathering experiments on CCs can also provide valuable support to the forthcoming asteroid sample return missions that will visit primitive asteroids expected to be rich in organic materials: OSIRIS-REx/NASA (launch planned in 2016) on B-type 101955 Bennu (Campins et al. 2010) and Hayabusa2/JAXA (launch at the end of 2014) on C-type 1999 JU3 (Takagi et al. 2011). Instruments on board the spacecrafts will collect spectra in the VIS-NIR range $(\sim 0.5-5 \mu \mathrm{m})$ to characterize the surface and to assess whether organic compounds are present. The spectral study of irradiated organics and meteorites in the laboratory can provide important indications of SpWe effects on the surface of asteroids and thus help select the best sampling site.

We have started a systematic experimental study on ion irradiation of CCs to better constrain the SpWe of dark objects. Testing several classes of CCs will give us a larger view of SpWe influence on the primitive dark asteroids. In a previous paper (Brunetto et al. 2014) we studied ion irradiation of CV Allende meteorite. Here we focus on the CM2 Murchison chondrite because of the well established link between the CM meteorites and the C-class asteroids (see, e.g., Burbine et al. 2002). This meteorite also has a matrix that has undergone a certain degree of aqueous alteration (Buseck \& Hua 1993) and the CM-class can be easily used to look for similarities with primitive asteroids (Lantz et al. 2013; Fornasier et al. 2014). Furthermore, we chose the Murchison meteorite to test the presence of the organic compounds after irradiation through the analysis of the aliphatic $\mathrm{CH}$ stretching bands at $3.4 \mu \mathrm{m}$, by analogy to what is observed on some C-class asteroids (e.g., 24 Themis, Campins et al. 2010).

\section{Experimental methods}

Several fragments of the Murchison meteorite were crushed into powder (unconstrained grain size) to prepare two pellets by pressing $\sim 0.1 \mathrm{~g}$ of meteorite on a $\mathrm{KBr}$ substrate. Ion irradiation of the pellets was performed on the SIDONIE platform (CSNSM, Orsay) with $\mathrm{He}^{+}$and $\mathrm{Ar}^{+}$at $40 \mathrm{keV}$. The following fluences were used (several spots allowed on one pellet, plus a central area left virgin): $1 \times 10^{16}$ and $3 \times 10^{16}$ ions $/ \mathrm{cm}^{2}$ for $\mathrm{He}^{+}$, and $2 \times 10^{15}$ and $6 \times 10^{15}$ ions $/ \mathrm{cm}^{2}$ for $\mathrm{Ar}^{+}$(see Brunetto et al. 2014 for details on the experimental setup and technique). We chose to prepare pellets because in our experimental setup samples are held vertically. Several authors have used pellets to irradiate meteorites (e.g., Hiroi et al. 2004; Vernazza et al. 2013, and references therein). Irradiation was performed in a vacuum chamber and we placed the samples under dry vacuum between irradiation sessions and reflectance measurements. The implication for the budget of adsorbed water is discussed in Sect. 4.2.

A system coupling infrared fibers and a grating spectrometer (Maya2000Pro, Ocean Optics) allowed us to perform bidirectionnal reflectance spectroscopy in the visible range (VIS-NIR, $0.425-1.025 \mu \mathrm{m})$ with a fixed collection angle $e=0^{\circ}$ and illumination angle $i=45^{\circ}$ or $i \approx 30^{\circ}$. Reference spectra were

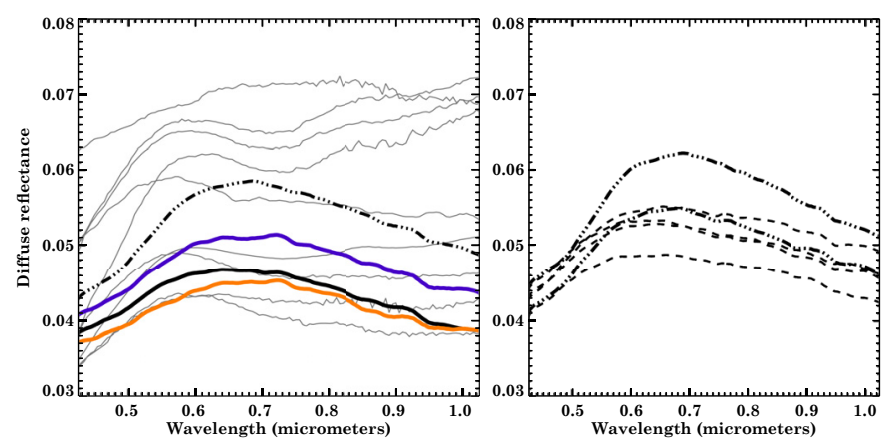

Fig. 1. Left: visible to near-infrared bidirectional $\left(i \approx 30^{\circ}\right.$ (dashed line) and $45^{\circ}$ (solid lines), $e=0^{\circ}$ ) diffuse reflectance spectra of one Murchison pellet before (black) and after (colored lines, only measured at $45^{\circ}$ ) irradiation with $3 \times 10^{16} \mathrm{He}^{+} / \mathrm{cm}^{2}$ (orange) and $6 \times 10^{15} \mathrm{Ar}^{+} / \mathrm{cm}^{2}$ (purple), compared with RELAB powder spectra (gray) as presented in Cloutis et al. $\left(2011 ; i=30^{\circ}, e=0^{\circ}\right)$. Right: visible to near-infrared bidirectional diffuse reflectance spectra (viewing geometry at $\approx 30^{\circ}$ ) on both pellets before irradiation to show surface heterogeneity of each sample.

acquired using a 99\% Spectralon standard (Labsphere) and the spectral resolution was fixed at $1 \mathrm{~nm}$. The SMIS (Spectroscopy and Microscopy in the Infrared using Synchrotron; Dumas et al. 2006) beamline at the synchrotron SOLEIL was used to perform near- to mid-infrared (NIR-MIR, 2-16 $\mu \mathrm{m}$ ) micro-analysis (focused beam of $15 \mu \mathrm{m}$, objective $\mathrm{x} 32$, confocal reflection). Reference spectra were collected on gold and the spectral resolution was $2 \mathrm{~cm}^{-1}$. Raman micro-spectroscopy was also performed at the SMIS beamline at SOLEIL using a $532 \mathrm{~nm}$ exciting laser radiation, and a power on the sample lower than $0.3 \mathrm{~mW}$. We used a spectral resolution of $4 \mathrm{~cm}^{-1}$ and a spatial resolution of about $1 \mu \mathrm{m}$, and we probed matrix areas (see Brunetto et al. 2014 for more details). In all spectral ranges, several spectra were acquired to account for the sample heterogeneity.

\section{Results}

\subsection{Visible-NIR range}

We present in Fig. 1 (left panel) the VIS-NIR spectra at $i \approx$ $30^{\circ}$ and $45^{\circ}$. The first angle can be used to compare our spectrum with data presented in Cloutis et al. (2011; from RELAB database, powder with different grain sizes, viewing geometry at $\left.30^{\circ}\right)$. The Murchison meteorite has a low albedo $(\sim 5 \%$ at $0.55 \mu \mathrm{m})$, close to what has been noticed by Johnson \& Fanale (1973), but the UV drop-off is slightly less marked for the $i=45^{\circ}$ measure. In our sample before irradiation we clearly see the phase angle effect: as expected reflectance at $i \approx 30^{\circ}$ is higher than that at $i=45^{\circ}$. The irradiation has little effect on the Murchison meteorite in this spectral range. There is a small drop in the reflectance for the helium irradiation (from 4.5 to $4.2 \%$ at $0.55 \mu \mathrm{m}$; less than $3 \%$ decrease over the whole VISNIR range), while there is an increase in the argon irradiation (albedo at $0.55 \mu \mathrm{m}$ goes to $4.8 \%$; $8 \%$ increase over the whole range), and almost no change in the spectral slope is observed for either ion. These changes are weaker than other variations owing to the viewing geometry and grain size effects (shown in Fig. 1 left panel compared with RELAB spectra). Spectra variations after irradiation are also smaller than variations due to the sample heterogeneity (Fig. 1 right panel). 


\subsection{NIR-MIR}

We present in Fig. 2 (upper panel) the NIR-MIR spectra (average of about 40 spectra for each area) before irradiation and after the highest fluence with both ions. We restrict our analysis to matrix areas to focus on the carbon-rich component. The broad $\mathrm{OH}$ stretching band is detected between $2.7 \mu \mathrm{m}$ and $3 \mu \mathrm{m}$ and the $\mathrm{H}_{2} \mathrm{O}$ bending band around $6 \mu \mathrm{m}$. These absorption bands can be attributed to aqueously altered minerals and/or to adsorbed water (see below). The Christiansen feature (indicating a rapid increase of the refractive index, Salisbury \& D'Aria 1989) before $9 \mu \mathrm{m}$ and the phyllosilicate peak near $10 \mu \mathrm{m}$ are clearly observed. This is consistent with a diffuse reflectance spectrum of Murchison meteorite obtained by Miyamoto (1987). In Fig. 2 (lower panel), we also present NIR-MIR spectra (average of about ten spectra for each area) before and after $2 \times 10^{15} \mathrm{Ar}^{+} / \mathrm{cm}^{2}$ and $1 \times 10^{16} \mathrm{He}^{+} / \mathrm{cm}^{2}$ irradiation on the second pellet. We observe the similar spectral features, plus the $\mathrm{CH}$ stretching bands near $3.4 \mu \mathrm{m}$. We can also see that in the $10 \mu \mathrm{m}$ region, the olivine band contribution is stronger in the second pellet than in the first one, while the phyllosilicate band is weaker. Cloutis et al. (2011) noticed that different subsamples of the Murchison meteorite can have unique spectral properties due to the meteorite heterogeneity.

The irradiation effects on the $\mathrm{OH}$ stretching band are shown in Fig. 3. We detect a broad asymmetric band with a minimum around $2.73 \mu \mathrm{m}$ before irradiation consistent with the Beck et al. (2010) and Miyamoto (1990) spectra. This band is similar to that observed on some C-class asteroids like 2 Pallas and 51 Nemausa (Rivkin et al. 2003). On the right side of the band a convex behavior can be seen centered around $2.90 \mu \mathrm{m}$ that becomes concave after irradiations. The band profile on the left side is also affected, as the $2.71 \mu \mathrm{m}$ feature (indicated with a gray line) disappears after irradiation. Furthermore, we observe a shift of the band: the minimum is located at $2.73 \mu \mathrm{m}$ before irradiation and goes to $2.76 \mu \mathrm{m}$ after the highest helium dose. The error on the band minimum detection is $\sim 0.005 \mu \mathrm{m}$ (spectral resolution at this wavelength is $\sim 0.0015 \mu \mathrm{m}$ ). This shift is larger than variations due to sample heterogeneity that are $\pm 0.02 \mu \mathrm{m}$. The $\mathrm{H}_{2} \mathrm{O}$ bending feature near $6 \mu \mathrm{m}$ is also shown in Fig. 3 where there is a simplification of the structure after irradiation. The right side of the $3.0 \mu \mathrm{m}$ band and the $6.0 \mu \mathrm{m}$ feature are similarly affected on the second pellet (Fig. 2 lower panel).

The NIR reflectance spectra of the second Murchison pellet show the presence of the aliphatic $\mathrm{CH}$ stretching band in this region (see Fig. 4). The band is actually the blend of at least four bands corresponding to the symmetric and antisymmetric $\mathrm{CH}_{2}$ and $\mathrm{CH}_{3}$ stretching modes (see, e.g., Dartois et al. 2004). The asymmetric modes (left component of the band) we detect on each pellet area represent about $1.5 \%$ depth (with respect to the continuum) and we do not see any effect on the band area either with $\mathrm{Ar}^{+}$or $\mathrm{He}^{+}$irradiation at any fluence; the area is about $1.80 \pm 0.03 \mathrm{~cm}^{-1}$, calculated after continuum removal over the range $3.30-3.55 \mu \mathrm{m}$, before and after irradiation (with the argon dose indicated in Fig. 4).

In the MIR range (see Fig. 5) a phyllosilicate peak is detected at $9.87 \pm 0.03 \mu \mathrm{m}$ (the error bars take into account the sample heterogeneity). This is in agreement with Beck et al. (2010) who observed that CM meteorites do not compare with known terrestrial serpentine in the $10 \mu \mathrm{m}$ region. This peak becomes less intense than the second maximum on its right (intensity goes from almost 0.01 in reflectance unit to less than 0.005 ), and it shifts towards longer wavelength while irradiation dose increases, to reach $9.94 \pm 0.02 \mu \mathrm{m}$ (error bars come
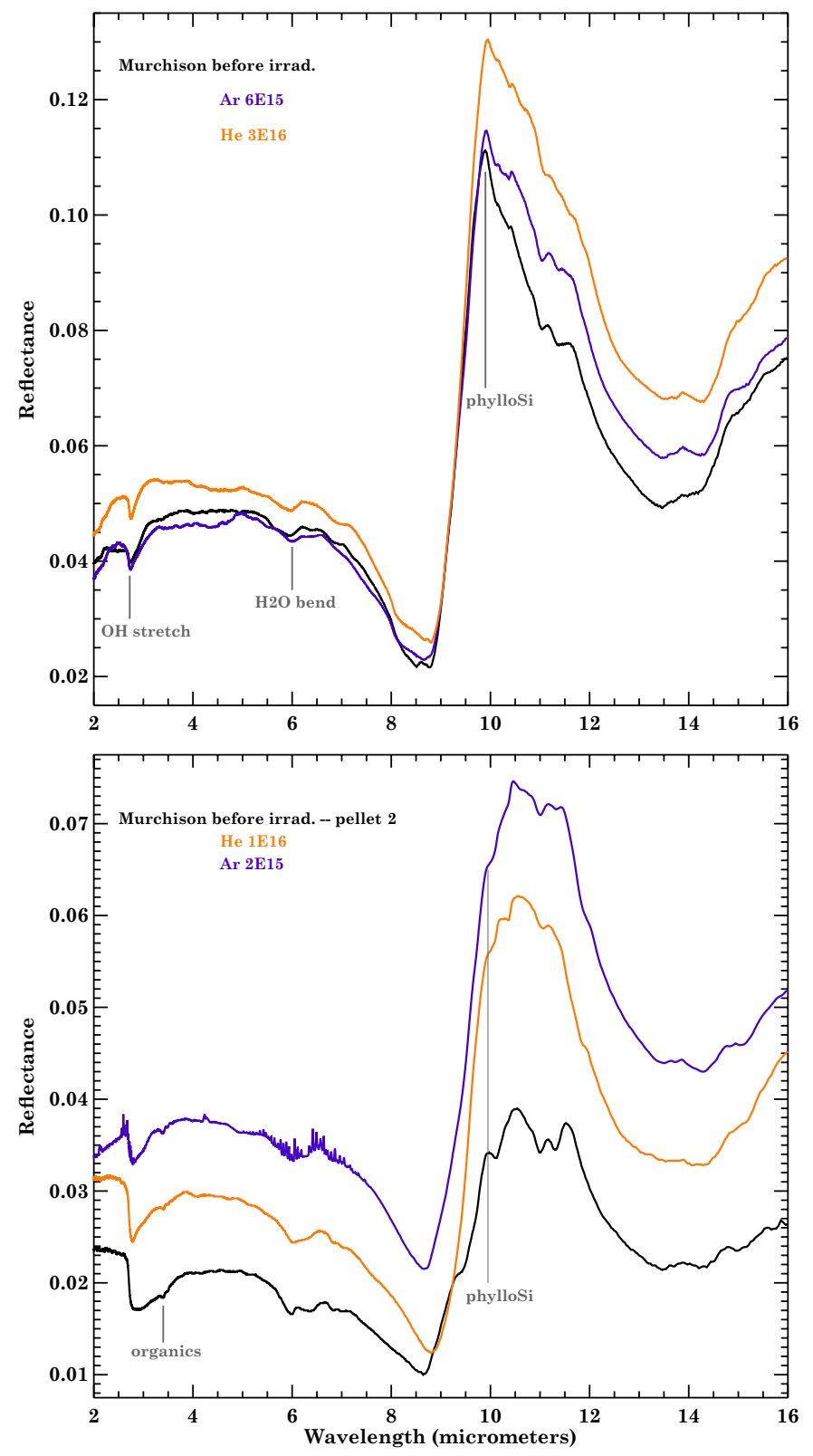

Fig. 2. Top: NIR-MIR confocal reflectance spectra of a non-irradiated area of the first Murchison pellet (black), and those irradiated at the highest fluence for both used ions (purple for argon and orange for helium). Bottom: same as before: virgin, $2 \times 10^{15} \mathrm{Ar}^{+} / \mathrm{cm}^{2}$ and $1 \times$ $10^{16} \mathrm{He}^{+} / \mathrm{cm}^{2}$ irradiated areas on the second pellet.

from sample heterogeneity) with the highest helium dose. The shift is larger than variations due to the pellet heterogeneity. Furthermore we detect a peak at $11.17 \mu \mathrm{m}$ corresponding to the olivine contribution reported by Beck et al. (2014) and Morlok et al. (2010), shifting also to $11.21 \mu \mathrm{m}$ after irradiation. Another peak is present at $11.65 \mu \mathrm{m}$, that could be associated to enstatite, and it shifts to $11.69 \mu \mathrm{m}$ and strongly decreases after irradiation (intensity goes from almost 0.005 in reflectance unit to less than 0.002). Two smaller peaks are seen at $10.16 \mu \mathrm{m}$ (not changing after irradiation) and $10.42 \mu \mathrm{m}$ going to $10.45 \mu \mathrm{m}$ after the highest helium dose. They are both attributed to olivine (Hamilton 2010). On the second pellet (see Fig. 2 lower panel), the phyllosilicate peak is seen at $9.95 \mu \mathrm{m}$ before irradiation and then disappears. The silicate peaks broaden after irradiation as 

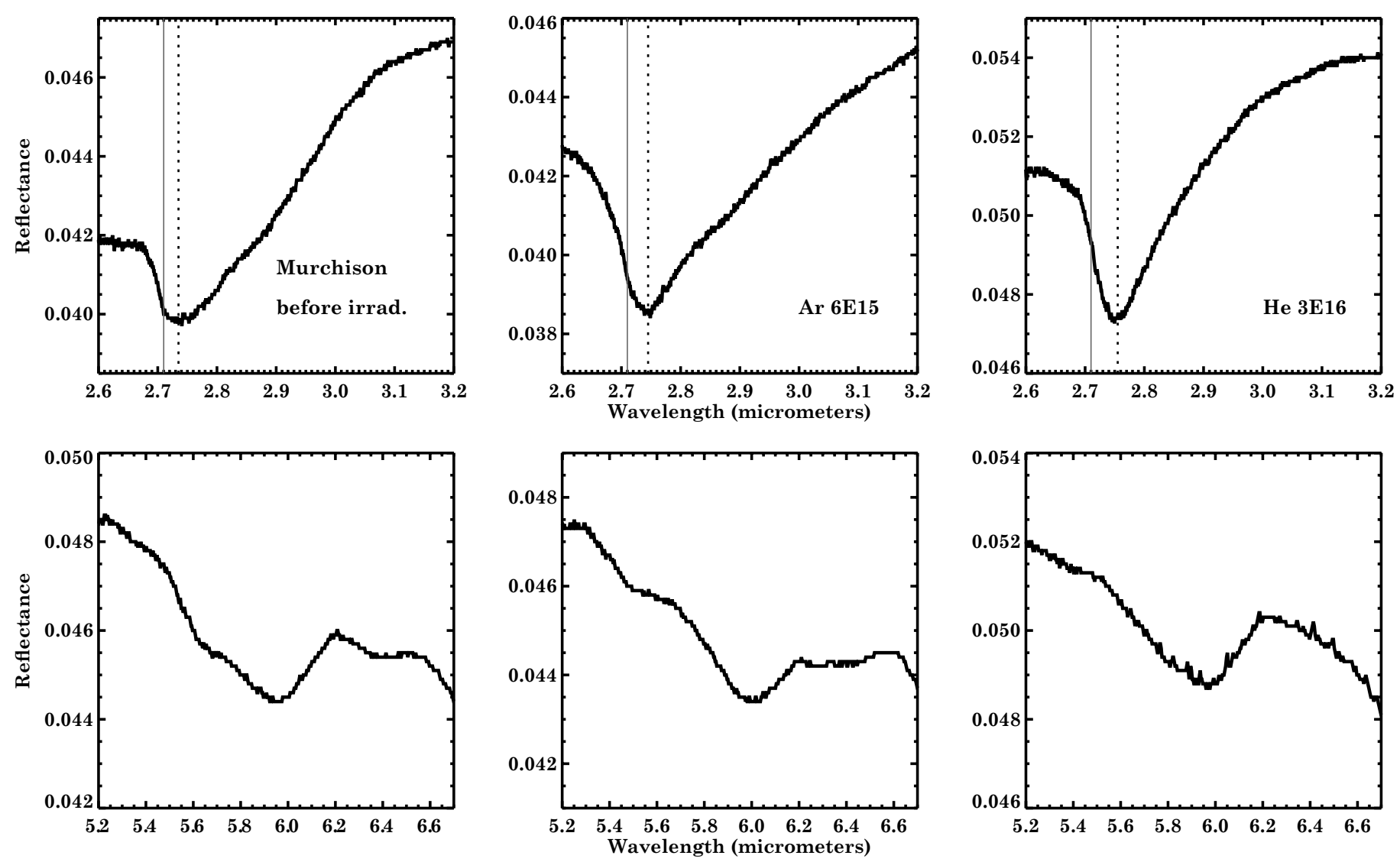

Fig. 3. NIR confocal reflectance spectra around aqueous alteration bands $\left(\mathrm{OH}\right.$-stretching on top, $\mathrm{H}_{2} \mathrm{O}$-bending on bottom) for the non-irradiated Murchison pellet area (left), and the highest fluence of irradiation for argon (central) and helium (right) ions. For the stretching mode: the gray line indicates the $2.71 \mu \mathrm{m}$ position on each plot, the dotted line indicates the band minimum shifting toward longer wavelength with increasing irradiation dose.

amorphization takes place (see, e.g., Brucato et al. 2004, and references therein).

\subsection{Raman}

In Fig. 6 we present a few Raman spectra for several irradiation doses and the unirradiated area. Raman spectroscopy is a nearsurface technique that is affected by the same light absorption issues described for the $3.4 \mu \mathrm{m}$ band (see below). The optical properties of the meteorite at the wavelength of the laser exciting radiation used here $(532 \mathrm{~nm})$ determine whether the probed depth is deeper or of the same order of magnitude as the irradiated layer, but in our Raman microscopic configuration the analyzed spot $(1 \mu \mathrm{m})$ is closer to the heterogeneity of the meteorite matrix than the IR case, and the probed depth thus strongly depends on the composition of the specific grains targeted by the laser (low-absorption vs. high-absorption grains, e.g., silicates vs. carbons). Since low- and high-absorption materials in the matrix are intimately mixed at a sub-wavelength scale, the probed layer will be comparable to or deeper than the ion penetration depth according to the layered configuration of the specific spot (i.e., whether carbon is on top or not). The consequence is that it is necessary to invoke a statistical approach to understand the effect of irradiation on the measured Raman spectra. We applied the same procedure described in detail by Brunetto et al. (2014). Here we show the most disordered component of each irradiated area. We clearly see the enlargement and displacement of carbon $D$ - and $G$-bands, similar to what has been observed in the case of Allende (Brunetto et al. 2014). See the full discussion in Brunetto et al. (2014); it is beyond the scope of the study here.

\section{Discussion}

\subsection{Organic materials}

The $\mathrm{CH}$ bonds are expected to be affected by ion irradiation processes. The destruction of aliphatic $\mathrm{CHs}$ and the corresponding loss of hydrogen has been extensively studied in the case of interstellar medium carbon-rich grains (see, e.g., Godard et al. 2011, and references therein). The efficiency of the aliphatic band destruction process depends on the stopping power. Godard et al. (2011) have shown that, in a regime of electronic energy loss (i.e., when the ion energy loss is essentially due to inelastic collisions with the material's electrons), the destruction cross section increases with $S_{\mathrm{e}}$ (the electronic stopping cross section, i.e., the electronic stopping power divided by the target density) following a power law with an index of $1.3 \pm 0.5$ (in a pure $\left(\mathrm{C}_{x} \mathrm{H}_{y}\right)_{n}$ material), but that for $S_{\mathrm{e}}<10 \mathrm{MeV} \mathrm{mg}^{-1} \mathrm{~cm}^{2}$ a significant number of $\mathrm{CH}$ bonds could be preserved ( $>30 \%$ of the original value). In our case, during the argon irradiation, the mechanism of energy loss is essentially dominated by elastic interactions between the ions and the target nuclei, so no prediction can be done with respect to the Godard et al. (2011) results (we can notice though that for this study, irradiation with $400 \mathrm{keV}$ Argon ions (i.e., higher proportion of elastic interactions) also results in the destruction of aliphatic $\mathrm{C}-\mathrm{H})$. In the case of helium irradiation $\left(S_{\mathrm{e}}=0.48 \mathrm{MeV} \mathrm{mg}^{-1} \mathrm{~cm}^{2}\right.$, a Stopping and Range 
C. Lantz et al.: Ion irradiation of the Murchison meteorite: Visible to mid-infrared spectroscopic results
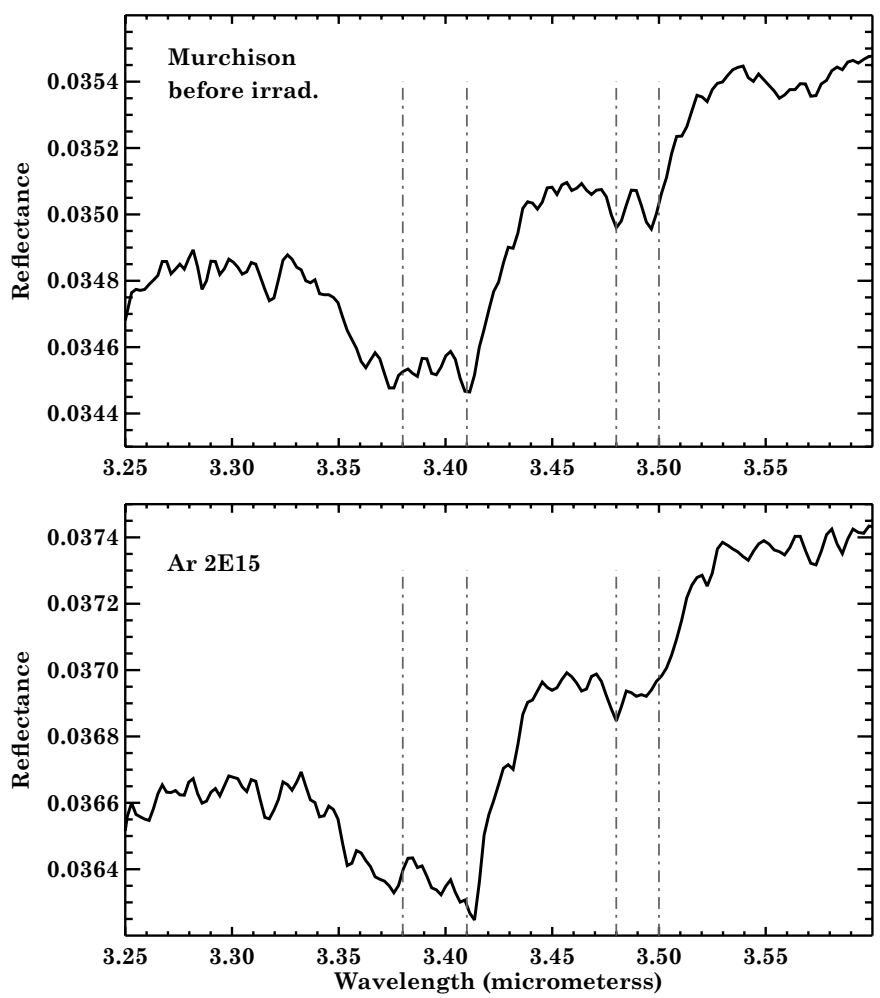

Fig. 4. NIR confocal reflectance spectra around the $\mathrm{CH}$ stretching bands for unirradiated Murchison (top) and after irradiation with $2 \times$ $10^{15} \mathrm{Ar}^{+} / \mathrm{cm}^{2}$ (bottom). We plotted vertical lines to indicate the expected positions (for transmittance measurements) of the antisymmetric 3.38 and $3.41 \mu \mathrm{m}$ and symmetric 3.48 and $3.50 \mu$ m modes of $\mathrm{CH}_{3}$ and $\mathrm{CH}_{2}$, respectively.

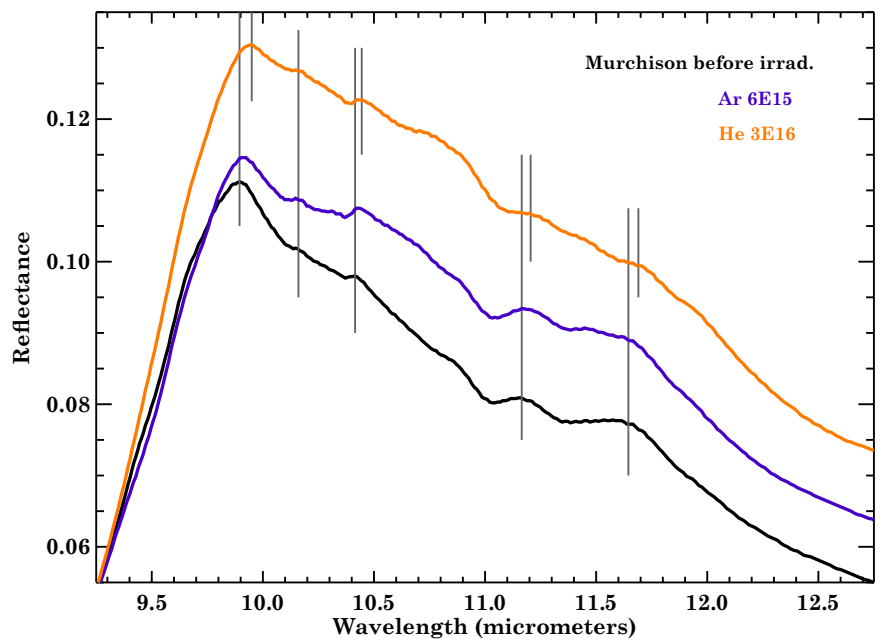

Fig. 5. MIR confocal reflectance spectra around the phyllosilicate peak at $10 \mu \mathrm{m}$. Vertical gray lines are plotted to guide the eye and indicate the band shift from the virgin spectrum (solid lines) to the $\mathrm{He}^{+}$irradiated spectrum (dashed).

of Ions in Matter simulation results, see Brunetto et al. 2014), at the beginning of the ion track the energy loss is essentially due to electronic interactions. Using the Godard et al. (2011) results, the destruction cross section corresponding to the helium $40 \mathrm{keV}$ irradiation would be $\sim 1 \times 10^{-16} \mathrm{~cm}^{2} /$ ion. This implies that at the highest fluence of $3 \times 10^{16} \mathrm{He}^{+} / \mathrm{cm}^{2}$ the $\mathrm{CH}$ band would essentially be close to its asymptotic value, which is lower
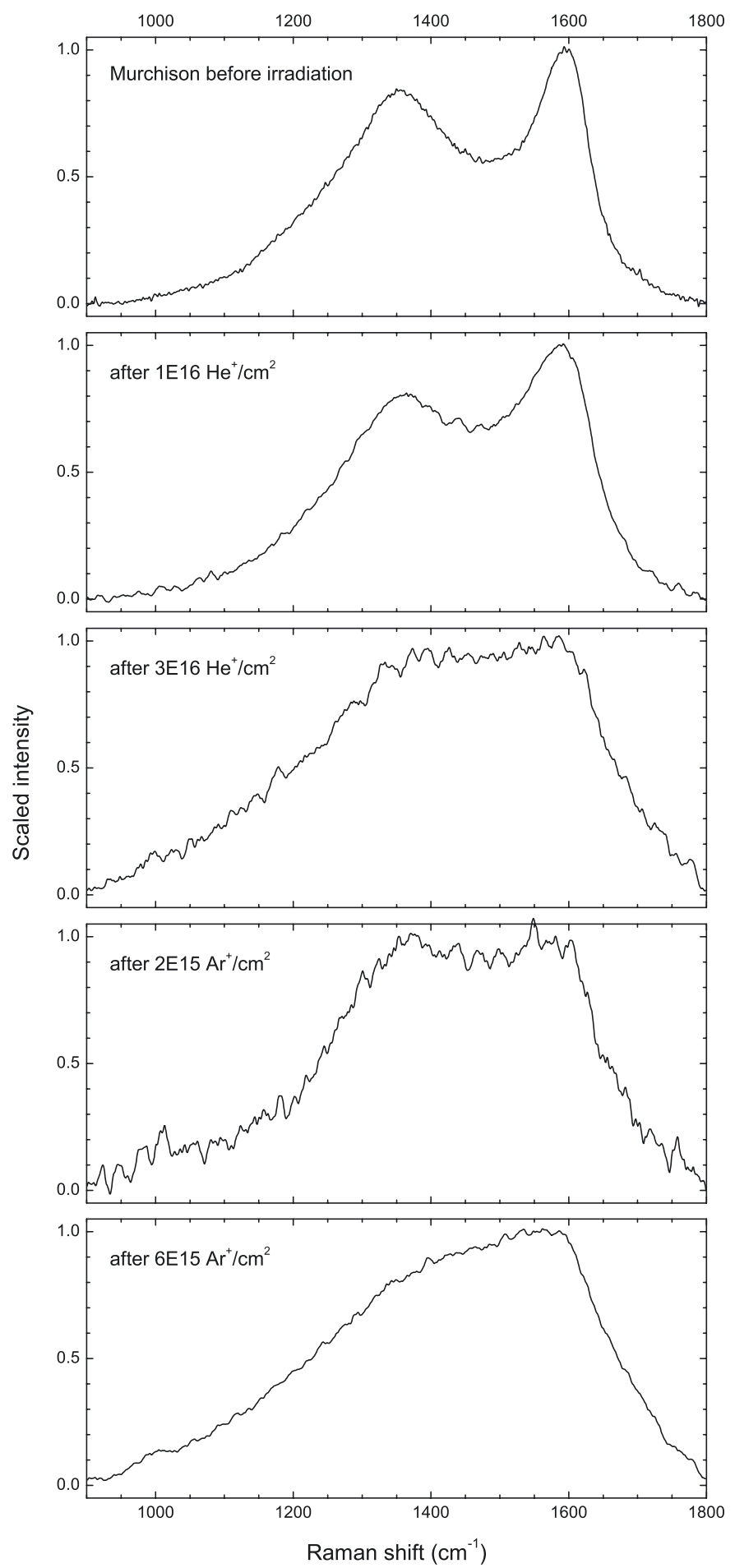

Fig. 6. Raman spectra of the unirradiated area (top) and for irradiated areas with $1 \times 10^{16}$ and $3 \times 10^{16} \mathrm{He}^{+}, 2 \times 10^{15}$ and $6 \times 10^{15} \mathrm{Ar}^{+}$(from top to bottom) ions $/ \mathrm{cm}^{2}$.

than $60-65 \%$ with respect to the initial abundance for the case of highly hydrogenated amorphous carbons.

Thus, if the reflected light in our Murchison irradiation experiment probed a surface depth smaller than or of the same order as the ion penetration depth, then a significant reduction of approximately a factor of two in the $\mathrm{CH}$ integrated area would be expected. However, no significant variations were observed (see Fig. 4), within the error bars, after helium irradiation or argon irradiation. We observed the same behavior in previous 
experiments on Allende (Brunetto et al. 2014). We conclude that the IR light at this wavelength probes a layer larger than the one affected by irradiation which is $40 \pm 15 \mathrm{~nm}$ and $300 \pm 80 \mathrm{~nm}$ for argon and helium ions, respectively - calculated on a simplified composition of CCs (Brunetto et al. 2014) using the SRIM code (Ziegler et al. 2010). A reasonable estimate of the photon penetration depth can be obtained using the imaginary index value calculated for the Tagish Lake meteorite $\left(5.5 \times 10^{-2}\right.$, Roush 2003). The depth in the sample from which the laser radiation emerges reduced to $50 \%$ of the incoming intensity would be $\sim 1 \mu \mathrm{m}$. Taking into account this discussion, we can conclude that the organic materials in the top layers are indeed affected by irradiation and that the $3.4 \mu \mathrm{m}$ photons probe a layer thicker than the ion penetration depth. One can imagine that irradiation with higher energy ions - as a simulation of solar flares, events from active regions, and/or cosmic rays - could lead to a modification of the $3.4 \mu \mathrm{m}$ band because these ions would have a larger penetration depth.

The fact that the organics in the very first layers are affected by irradiation is confirmed by the Raman analysis (most sensitive to the aromatic component but still allows organics to be probed): the aromatic carbon structure is affected by irradiation leading to an amorphization of this aromatic structure. Different degrees of de-ordering are produced as a function of dose, to finally end with a highly disordered carbon. These modifications have been previously reported to scale with the nuclear elastic dose in the case of terrestrial carbons (e.g., Brunetto et al. 2004, 2009 , and references therein), and this is confirmed also for extraterrestrial aromatic carbons (Brunetto et al. 2014). The results on Murchison (Fig. 6) agree with these previous studies.

Another interpretation for the non-destruction of the $3.4 \mu \mathrm{m}$ band in view of the Godard et al. (2011) results would be that the concentration of hydrogen bonded to aliphatic carbon is too low within the meteorite sample so that the free $\mathrm{H}$ atoms liberated by the destruction of aliphatic $\mathrm{C}-\mathrm{H}$ bonding by the impinging ions are too far from each other to recombine. The dehydrogenation of the material as caused by the molecular $\mathrm{H}_{2}$ formation would have already reached the asymptotic regime and no modification of the band could be seen. But the $\mathrm{H} / \mathrm{C}$ ratios in the Godard et al. (2011) experiments are about 1, while in the Murchison meteorite the ratio is about 0.7 within the Insoluble Organic Matter (Hayatsu et al. 1980) and 1.55 within the Soluble Organic Matter (Schmitt-Kopplin et al. 2010). The hydrogen concentration within the Murchison is of the same order as within the Godard et al. (2011) samples so we could expect a spectral decrease of the aliphatic organic band. This is not the case.

\subsection{Phyllosilicates}

In the Murchison VIS spectra (Fig. 1) we do not see the $0.7 \mu \mathrm{m}$ band that is associated with aqueous alteration processes and the presence of phyllosilicates in the visible range (Vilas \& Gaffey 1989). It is interesting to note that half of RELAB spectra (gray thin lines in Fig. 1) do not show this absorption band either, whereas the $3.0 \mu \mathrm{m}$ band is detected (Fig. 3). This is not a surprising result as the $0.7 \mu \mathrm{m}$ band is fainter than the $3.0 \mu \mathrm{m}$ one; it has been shown that the $3.0 \mu \mathrm{m}$ band is almost always present when the $0.7 \mu \mathrm{m}$ one is detected, while the reverse statement is not true (Vilas 1994; Howell et al. 2011; Takir \& Emery 2012; Fornasier et al. 2014; McAdam et al. 2015).

The irradiation process leads to a phyllosilicate contribution removal (chrysotile-looking feature in Fig. 3) in the $2.7 \mu \mathrm{m}$ feature and/or to a shift of the band center towards the Ferich spectral region of serpentine. We note that $\mathrm{CM}$ meteorites are mainly made of $\mathrm{Mg}$-serpentine and Fe-cronstedtite (Howard et al. 2009) and show a wide range of $\mathrm{Fe} / \mathrm{Mg}$ ratios (Tomeoka et al. 1989). However, it does not look like terrestrial minerals (e.g., chrysotile), and we talk about proto-serpentine below.

Furthermore, we notice a band profile modification on the right side of the wing, possibly owing to a removal of the adsorbed water component. Beck et al. (2010) and Takir et al. (2013) showed that measurements at ambiant conditions are biased because of adsorbed water and that this contribution can be efficiently removed thanks to heating experiments and vacuum exposure. The Murchison spectra were measured ex situ and adsorbed terrestrial water molecules are present in the sample, even if we placed the pellets under dry vacuum conditions between irradiation sessions and reflectance measurements preventing a long term adsorption on the sample surface. Indeed, our spectrum of virgin Murchison has a wing on the right side of the band stopping near $3.1 \mu \mathrm{m}$ that is less broad and intense than that measured under ambient conditions by Beck et al. (2010) on the Boriskino meteorite (CM2) where the wing goes to almost $4 \mu \mathrm{m}$. The Cold Bokkeveld meteorite (CM2) measured by Takir et al. (2013) has the same behavior. The $6 \mu \mathrm{m}$ band is also expected to reduce upon heating (Miyamoto 1990). Here we only see a simplification of the feature, but the intensity of the band in Miyamoto (1990) is much higher than that measured here (Fig. 3) and do not totally disappear even with increasing temperature. In other words, our experimental procedure seems to have ensured a relatively small amount of adsorbed water compared to previous published spectra under ambiant conditions. The modifications we observe in the band profile (see Fig. 3) could therefore be due to the irradiation process.

In the MIR range, the irradiation decreased the intensity of the phyllosilicate peak near $10 \mu \mathrm{m}$ and a shift of the peak toward longer wavelength is observed. The two strongest olivine peaks also undergo this band shift; this effect has already been described on Tagish Lake (Vernazza et al. 2013) and Allende (Brunetto et al. 2014). The latter discussed the shift amplitude as a function of the peak intensity, which is also observed here: the stronger the band intensity, the bigger the band shift. We note that these IR silicate bands are intense (thanks to a high refractive index) and are due to surface scattering.

The spectral signatures move from a $\mathrm{Mg}$-rich region to a $\mathrm{Fe}-$ rich one. As proposed by Brunetto et al. (2014), this effect could be due to a preferential $\mathrm{Mg}$ loss within minerals (in nesosilicates, olivine, as well as in phyllosilicates), that could be attributed to differential sputtering and/or amorphization of Mg-rich materials. Indeed, there is a differential sputtering of atoms near the surface; the lighter $\mathrm{Mg}$ is more easily sputtered backward than the less volatile Fe (Hapke et al. 1975). The amorphization of olivine after $\mathrm{He}^{+}$irradiation at the same energy used in this study (10-50 keV) has been underlined by Demyk et al. (2001) and Carrez et al. (2002), accompanied with $\mathrm{Mg} / \mathrm{Si}$ ratio decrease. The same result was obtained for a study on pyroxenes (Demyk et al. 2004), including enstatite. The band shift seen here is much less important in the $\mathrm{Ar}^{+}$irradiated spectrum (e.g., for the first peak in Fig. 5; the shift is almost $0.06 \mu \mathrm{m}$ in the helium case, while about $0.02 \mu \mathrm{m}$ in the argon case) and this can be explained by the penetration depth of the argon ions $(\sim 40 \mathrm{~nm})$ that affect a smaller layer than $\mathrm{He}^{+}(\sim 300 \mathrm{~nm})$. In the case of target irradiated by $\mathrm{Ar}^{+}$, IR photons mostly probe an unirradiated volume and the irradiation effects are less visible (see discussion in Brunetto et al. 2014). In the Carrez et al. (2002) paper, the authors also invoked a reduction process resulting in the formation of nanophase reduced iron $(\mathrm{npFe} 0)$ that can explain the surface enrichment in Fe. 
C. Lantz et al.: Ion irradiation of the Murchison meteorite: Visible to mid-infrared spectroscopic results
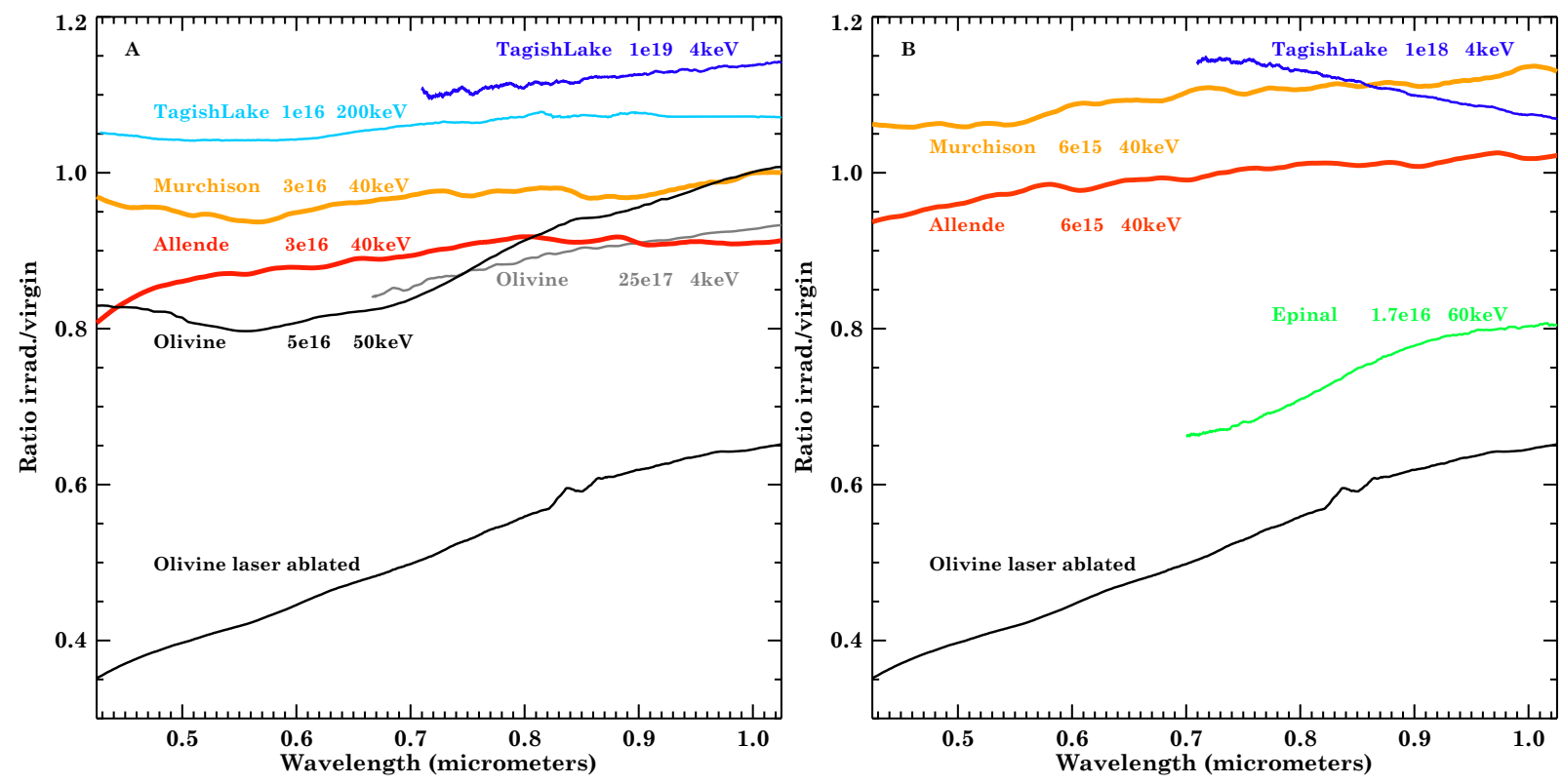

Fig. 7. Ratio plots for different meteorites and minerals: irradiated spectrum divided by the virgin spectrum. Irradiations are performed with $\mathrm{He}^{+}$ (panel A)) and $\mathrm{Ar}^{+}$(panel B)) ions. Tagish Lake spectra are from Vernazza et al. (2013). Olivine data, black and gray respectively, from Fu et al. (2012) and Loeffler et al. (2009). OC Epinal meteorite and terrestrial olivine laser ablated spectra come from Brunetto et al. (2006, 2007).

\subsection{Comparison with previous irradiations in the visible range}

Few carbonaceous chondrites have been studied using ion irradiation laboratory experiments. We present here the results obtained for Murchison, Tagish Lake, and Allende that all belong to the class of carbonaceous chondrites, but have distinct characteristics in terms of hydrothermal alteration on their respective parent bodies (e.g., Brearley \& Jones 1998). Murchison is a typical CM2 chondrite containing small $(\sim 200 \mu \mathrm{m})$ chondrules and refractory inclusions, abundant fine-grained matrix ( $\sim 70 \mathrm{vol} \%)$, and abundant hydrated minerals. It contains about $2 \mathrm{wt} \%$ of carbonaceous matter (Kerridge 1985), mostly in the form of insoluble organic matter, but the soluble part contains a large number of organic compounds (e.g., Schmitt-Kopplin et al. 2010, and references therein). Tagish lake is a C2 ungrouped carbonaceous chondrite that contains chondrules, abundant hydrated minerals, and fine-grained matrix. It does not fit in a well-established C2 group, as it contains two different lithologies (carbonaterich and carbonate-poor) with varying degrees of aqueous alteration (e.g., Zolensky et al. 2002). Tagish lake contains about $5.5 \mathrm{wt} \%$ of carbonaceous matter (Grady et al. 2002). Allende is a CV3 carbonaceous chondrite belonging to the oxidized subgroup, showing low degrees of aqueous alteration, and unequilibrated mineral assemblages (Rubin \& Wasson 1987). It contains large (mm-sized) chondrules, large refractory inclusions, and abundant matrix $(\sim 40 \mathrm{vol} \%)$. It contains only a small amount ( $\sim 0.3 \mathrm{wt} \%)$ of carbonaceous matter (Kerridge 1985).

Contradictory results are observed for the irradiation of these carbonaceus chondrites showing both reddening and blueing effects. In Fig. 7 we present two ratio plots (irradiated sample spectrum divided by the virgin sample, under the same viewing geometry) for ion irradiated carbonaceous chondrites (helium in the left panel, argon in the right panel), one ordinary chondrite and olivines recorded by this study or other groups. When comparing data from different laboratories, one can separate the sample preparation effect from the weathering effect by performing the ratio between spectra of altered and unaltered meteorites. As has been shown by Brunetto et al. (2006), the spectral ratio is mainly sensitive to the alteration effects, and has a weaker dependence on sample preparation.

When compared to Allende and olivines, Murchison shows little variation after irradiation in the $\mathrm{He}^{+}$case (see panel A): the spectrum remains relatively flat and the albedo decreases by $2.5 \%$. A linear fit in the $0.44-1.0 \mu \mathrm{m}$ range gives a ratio slope of $0.078 \mu \mathrm{m}^{-1}$. We observe a weak change in the slope after $\mathrm{Ar}^{+}$irradiation (see panel B): it rises to $0.13 \mu \mathrm{m}^{-1}$ within that range. A similar slope variation is obtained on the Allende sample analyzed by Brunetto et al. (2014) for both ions: this meteorite clearly reddens. Lazzarin et al. (2006) also noticed this reddening after $\mathrm{Ar}^{++}$irradiation at higher energy (400 keV).

In the case of Tagish Lake meteorite, we include results from two experiments with different energies (Vernazza et al. 2013). The bright blue line (panel A, greatest energy) is flat overall with a ratio slope of $0.09 \mu \mathrm{m}^{-1}$. A smaller energy, but bigger fluence of $\mathrm{He}^{+}$ions (dark blue line) shows a weak reddening. A reverse trend is observed with $\mathrm{Ar}^{+}$ions: a strong blueing is seen $\left(-0.28 \mu \mathrm{m}^{-1}\right.$ in the $0.72-1.0 \mu \mathrm{m}$ range $)$.

The behaviors of terrestrial olivine and Epinal OC meteorite are well known (Fu et al. 2012; Loeffler et al. 2009; Brunetto et al. 2006); a strong reddening is observed (at least $0.50 \mu \mathrm{m}^{-1}$ for the ratio slope) whatever the ion used, and even stronger in the case of laser ablation experiments simulating micrometeorite bombardment (Brunetto et al. 2007). Furthermore, all the olivine and Epinal samples clearly darken, while we cannot establish a general trend for carbonaceous chondrites.

The results are summarized in Fig. 8 where we present the value of the ratio at $0.72 \mu \mathrm{m}$ (left panel) and the slope (right panel) calculated over the range $0.44-1.0 \mu \mathrm{m}$ (or a smaller range starting at $0.72 \mu \mathrm{m}$ when available) as a function of the initial albedo before irradiation, for data shown in the previous figure. We also show on both slope and albedo the range of variations due to sample heterogeneity on the Murchison meteorite (obtained from Fig. 1 right panel). One can see that the weathering effects on this specific carbonaceous chondrite are of the order of variations due to heterogeneity. We also added data points 

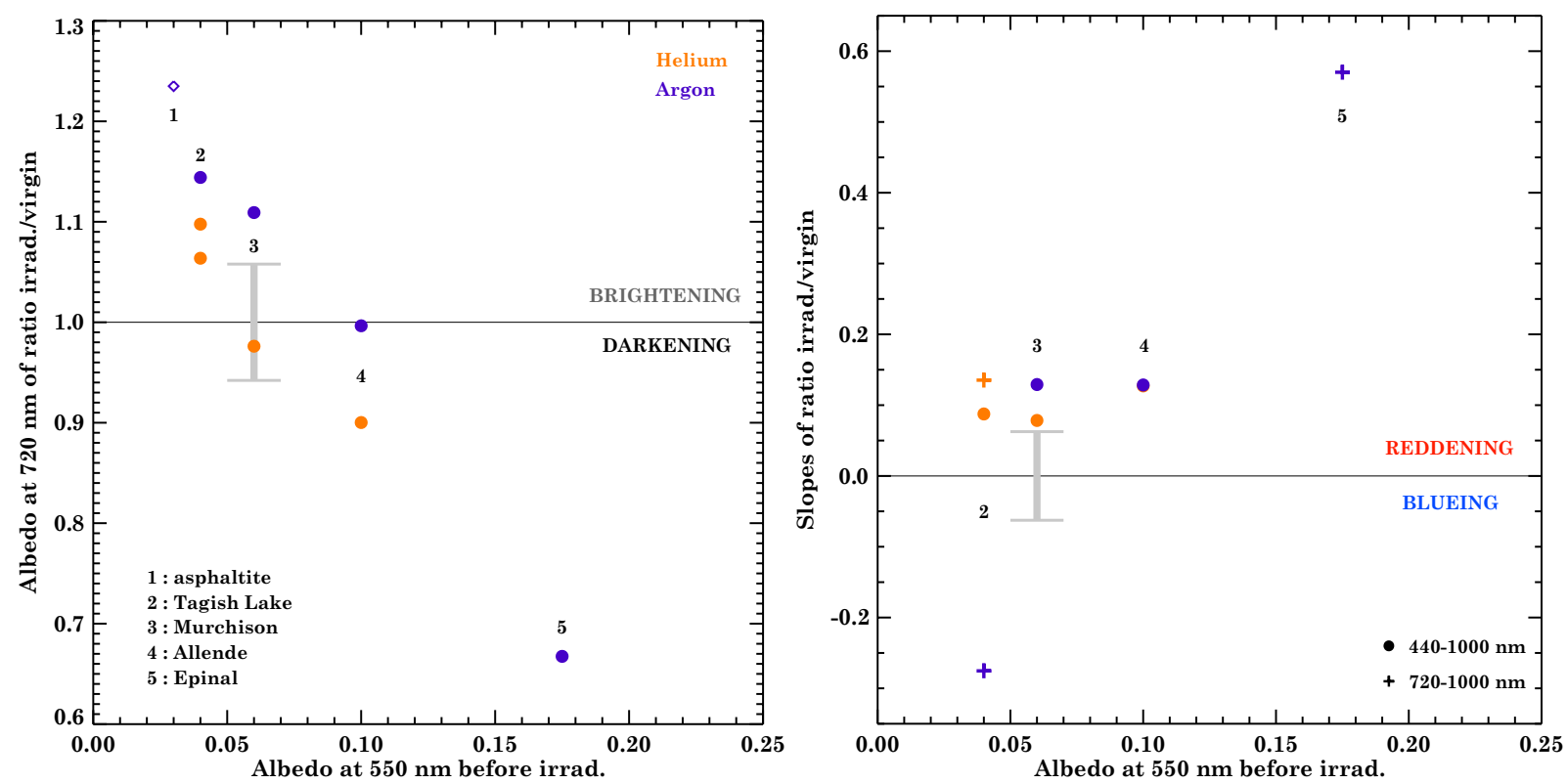

Fig. 8. Value of reflectance at $0.72 \mu \mathrm{m}$ (left panel) and spectral slopes (right panel) of the Fig. 7 ratios: virgin sample over irradiated sample. The orange dots are for irradiations with $\mathrm{He}^{+}$ions, purple is used for $\mathrm{Ar}^{+}$ions. The spectral slope is calculated using a linear fit on the whole range $(0.44-1.0 \mu \mathrm{m}$, dots $)$ when available or on a restricted area $(0.72-1.0 \mu \mathrm{m},+$ signs $)$ otherwise. The gray error bar in each panel indicates the variations due to our Murchison sample heterogeneity.

for an asphaltite sample from Moroz et al. (2004a) where a clear brightening of the surface was observed (see Fig. 8 left panel) after irradiation with $60 \mathrm{keV} \mathrm{Ar}^{++}$(fluence of $1.5 \times 10^{15} \mathrm{ions} / \mathrm{cm}^{2}$ ). A strong blueing was also a result of this experiment (not shown in the right panel of Fig. 8 because out of the range presented in the figure). The reddening and darkening trend observed on silicate-rich surfaces is not valid for all carbonaceous chondrites. From Fig. 8, it is clear that the spectral modifications after irradiation are a function of the initial albedo. Interestingly, the Tagish Lake meteorite represents the end-point of this transition from reddening to blueing, and from darkening to brightening. Among the samples reported in Fig. 8, Tagish Lake is the darkest and the richest in carbon.

\section{Conclusions}

In this paper we presented reflectance spectra of the CM2 Murchison meteorite probed by visible and infrared spectroscopy. We studied ion irradiation effects as a simulation of the solar wind bombardment of asteroid surfaces.

- The variations we observe within the visible range for irradiated Murchison are smaller than well-known viewing geometry, grain sizes, or sample preparation effects. We observe a reddening effect smaller than that observed in our previous experiment on Allende chondrite (Brunetto et al. 2014) and a light brightening (opposite effect for Allende) when taking into account both $\mathrm{He}^{+}$and $\mathrm{Ar}^{+}$ion results. The comparison of this work with the data presented in Brunetto et al. (2014) suggest that a relationship exists between the spectral variations observed and the initial composition.

- We do not see changes in the strength/profile of organic bands at $3.4 \mu \mathrm{m}$, probably because in this wavelength range, the reflectance probes a layer larger than the irradiated one and the effects of irradiation on this faint band are hidden in the volume. Raman measurements clearly show that the IOM (and probably SOM too) experienced chemical and structural modifications under irradiation.
- We observe a simplification of the features near 3 and $6 \mu \mathrm{m}$ related to adsorbed water after irradiation process.

- We noticed shifts of the phyllosilicate and olivine bands near 2.7 and $10 \mu \mathrm{m}$ toward the Fe-rich spectral region suggesting a loss of the element $\mathrm{Mg}$, probably due to a preferential sputtering of $\mathrm{Mg}$ and/or amorphization of $\mathrm{Mg}$-rich materials. These results cannot confirm the presence of npFe0, but do not disagree with the forming mechanism (Hapke 2001).

We observed that the spectral modifications in the visible range after irradiation are a function of the initial albedo. More experiments will be necessary to elucidate the role of the initial composition and carbon content on the spectral trend. Considering different samples of CCs within a systematic study will help us to build a strong SpWe model on primitive material in order to interpret the surface evolution of dark asteroids.

Acknowledgements. We warmly thank the Natural History Museum of Vienna and D. Cruikshank (NASA Ames) for providing us with the meteorite samples. We are grateful to X. Fu, D. Fulvio and M. Loeffler for sharing data. We thank D. Baklouti, P. Beck, P. Dumas, S. Fornasier, F. Jamme and C. Sandt for help and useful discussions. We also thank $\mathrm{H}$. Campins and G. Libourel for their reviews and helpful comments. This research is part of a joint IASCSNSM project (INGMAR) and it has been funded by the French national program "Programme National de Planétologie" (PNP), by the Faculté des Sciences d'Orsay, Université Paris-Sud ("Attractivité 2012"), by the French "Agence Nationale de la Recherche" (contract ANR-11-BS56-0026, OGRESSE), and by the P2IO LabEx (ANR-10-LABX-0038) in the framework "Investissements d'Avenir" (ANR-11-IDEX-0003-01) managed by the French National Research Agency (ANR). C.L. and M.A.B. acknowledge the funding of the French national space agency "Centre National d'Études Spatiales" (CNES).

\section{References}

Beck, P., Quirico, E., Montes-Hernandez, G., et al. 2010, Geochim. Cosmochim. Acta, 74, 4881

Beck, P., Garenne, A., Quirico, E., et al. 2014, Icarus, 229, 263

Brearley, A. J., \& Jones, R. H. 1998, Rev. Min. Geochem., 36, 3

Brucato, J. R., Strazzulla, G., Baratta, G., \& Colangeli, L. 2004, A\&A, 413, 395

Brunetto, R., Baratta, G. A., \& Strazzulla, G. 2004, J. Appl. Phys., 96, 380

Brunetto, R., Vernazza, P., Marchi, S., et al. 2006, Icarus, 184, 327

Brunetto, R., Roush, T. L., Marra, A. C., \& Orofino, V. 2007, Icarus, 191, 381 
Brunetto, R., Pino, T., Dartois, E., et al. 2009, Icarus, 200, 323

Brunetto, R., Lantz, C., Ledu, D., et al. 2014, Icarus, 237, 278

Burbine, T. H., McCoy, T. J., Meibom, A., Gladman, B., \& Keil, K. 2002, Asteroids III (Tucson: University of Arizona Press), 653

Buseck, P. R., \& Hua, X. 1993, Ann. Rev. Earth Planet. Sci., 21, 255

Campins, H., Hargrove, K., Pinilla-Alonso, N., et al. 2010a, Nature, 464, 1320

Campins, H., Morbidelli, A., Tsiganis, K., et al. 2010b, ApJ, 721, L53

Carrez, P., Demyk, K., Cordier, P., et al. 2002, Meteor. Planet. Sci., 37, 1599

Clark, B. E., Lucey, P., Helfenstein, P., et al. 2001, Meteor. Planet. Sci., 36, 1617

Clark, B. E., Hapke, B., Pieters, C., \& Britt, D. 2002, Asteroids III (Tucson: University of Arizona Press), 585

Cloutis, E. A., Hudon, P., Hiroi, T., Gaffey, M. J., \& Mann, P. 2011, Icarus, 216, 309

Dartois, E., Muñoz-Caro, G., Deboffle, D., \& d'Hendecourt, L. 2004, A\&A, 423, L33

DeMeo, F. E., \& Carry, B. 2014, Nature, 505, 629

Demyk, K., Carrez, P., Leroux, H., et al. 2001, A\&A, 368, L38

Demyk, K., d'Hendecourt, L., Leroux, H., Jones, A. P., \& Borg, J. 2004, A\&A, 420,233

Fornasier, S., Lantz, C., Barucci, M. A., \& Lazzarin, M. 2014, Icarus, 233, 163

Fu, X., Zou, Y., Zheng, Y., \& Ouyang, Z. 2012, Icarus, 219, 630

Gaffey, M. J., Cloutis, E. A., Kelley, M. S., \& Reed, K. L. 2002 (Tucson: University of Arizona Press), Asteroids III, 183

Godard, M., Féraud, G., Chabot, M., et al. 2011, A\&A, 529, A146

Grady, M. 2000, Catalogue of Meteorites: with Special Reference to Those Represented in the Collection of the Natural History Museum, London (Cambridge University Press)

Grady, M. M., Verchovsky, A. B., Franchi, I. A., Wright, I. P., \& Pillinger, C. T. 2002, Meteor. Planet. Sci., 37, 713

Hamilton, V. E. 2010, Chemie der Erde/Geochemistry, 70, 7

Hapke, B. 2001, J. Geophys. Res., 106, 10039

Hapke, B., Cassidy, W., \& Wells, E. 1975, Moon, 13, 339

Hayatsu, R., Winans, R. E., Scott, R. G., et al. 1980, Science, 207, 1202

Hiroi, T., Pieters, C. M., Rutherford, M. J., et al. 2004, in Lunar and Planetary Sci. Conf. 35, eds. S. Mackwell, \& E. Stansbery, 1616

Hiroi, T., Sasaki, S., Misu, T., \& Nakamura, T. 2013, in Lunar and Planetary Inst. Techn. Rep., 44, 1276

Howard, K. T., Benedix, G. K., Bland, P. A., \& Cressey, G. 2009, Geochim. Cosmochim. Acta, 73, 4576

Howell, E. S., Rivkin, A. S., Vilas, F., et al. 2011, in EPSC-DPS Joint Meet. held 2-7 October in Nantes, France, 637

Ishiguro, M., Hiroi, T., Tholen, D. J., et al. 2007, Meteor. Planet. Sci., 42, 1791

Johnson, T. V., \& Fanale, F. P. 1973, J. Geophys. Res., 78, 8507

Kerridge, J. F. 1985, Geochim. Cosmochim. Acta, 49, 1707
Lantz, C., Clark, B. E., Barucci, M. A., \& Lauretta, D. S. 2013, A\&A, 554, A138

Lazzarin, M., Marchi, S., Moroz, L. V., et al. 2006, ApJ, 647, L179

Loeffler, M. J., Dukes, C. A., \& Baragiola, R. A. 2009, J. Geophys. Res., 114, 3003

McAdam, M., Sunshine, J., Howard, K., \& McCoy, T. 2015, Icarus, 245, 320

Miyamoto, M. 1987, Icarus, 70, 146

Miyamoto, M. 1990, in Lunar and Planetary Sci. Conf., 21, 797

Morlok, A., Koike, C., Tomioka, N., Mann, I., \& Tomeoka, K. 2010, Icarus, 207, 45

Moroz, L., Baratta, G., Strazzulla, G., et al. 2004a, Icarus, 170, 214

Moroz, L. V., Hiroi, T., Shingareva, T. V., et al. 2004b, in Lunar and Planetary Sci. Conf. 35, eds. S. Mackwell \& E. Stansbery, 1279

Nesvorný, D., Jedicke, R., Whiteley, R. J., \& Ivezić, Ž. 2005, Icarus, 173, 132

Noble, S. K., Pieters, C. M., \& Keller, L. P. 2007, Icarus, 192, 629

Noguchi, T., Nakamura, T., Kimura, M., et al. 2011, Science, 333, 1121

Pieters, C. M., Taylor, L. A., Noble, S. K., et al. 2000, Meteor. Planet. Sci., 35, 1101

Rivkin, A. S., Davies, J. K., Johnson, J. R., et al. 2003, Meteor. Planet. Sci., 38, 1383

Roush, T. L. 2003, Meteor. Planet. Sci., 38, 419

Rubin, A. E., \& Wasson, J. T. 1987, Geochim. Cosmochim. Acta, 51, 1923

Salisbury, J. W., \& D'Aria, D. M. 1989, in Lunar and Planetary Inst. Technical Report, Vol. 20, Lunar and Planetary Sci. Conf., 940

Schmitt-Kopplin, P., Gabelica, Z., Gougeon, R. D., et al. 2010, Proc. Nat. Acad. Sci., 107, 2763

Shingareva, T. V., Basilevsky, A. T., Fisenko, A. V., et al. 2003, in Lunar and Planetary Sci. Conf. 34, eds. S. Mackwell, \& E. Stansbery, 1321

Shingareva, T. V., Basilevsky, A. T., Fisenko, A. V., Semjonova, L. F., \& Korotaeva, N. N. 2004, in Lunar and Planetary Sci. Conf. 35, eds. S. Mackwell, \& E. Stansbery, 1137

Takagi, Y., Yoshikawa, M., Abe, M., et al. 2011, AGU Fall Meet. Abstr.

Takir, D., \& Emery, J. P. 2012, Icarus, 219, 641

Takir, D., Emery, J. P., McSween, H. Y., et al. 2013, Meteor. Planet. Sci., 48, 1618

Tomeoka, K., McSween, Jr., H. Y., \& Buseck, P. R. 1989, Antarctic Meteorite Research, 2, 221

Vernazza, P., Fulvio, D., Brunetto, R., et al. 2013, Icarus, 225, 517

Vilas, F. 1994, Icarus, 111, 456

Vilas, F., \& Gaffey, M. J. 1989, Science, 246, 790

Ziegler, J. F., Ziegler, M. D., \& Biersack, J. P. 2010, Nucl. Instrum. Methods Phys. Res. B, 268, 1818

Zolensky, M. E., Nakamura, K., Gounelle, M., et al. 2002, Meteor. Planet. Sci., 37, 737 